\title{
Aspects de la complémentation en FLE chez des apprenants hellénophones
}

\author{
Fryni Kakoyianni-Doa ${ }^{1 *}$, Dora Loizidou ${ }^{1}$, et Monique Monville-Burston ${ }^{2}$ \\ ${ }^{1}$ Département d'Études françaises et européennes, Université de Chypre, PO Box \\ 20537, 1678, Nicosie, Chypre \\ ${ }^{2}$ Language Centre, Cyprus University of Technology, PO Box 50329, 3036 Limassol, \\ Chypre
}

\begin{abstract}
Résumé. Dans les grammaires/manuels du FLE, les sections consacrées aux complétives (autres qu'interrogatives) insistent particulièrement sur trois aspects de ce secteur de la grammaire française : l'emploi du mode (indicatif/subjonctif) dans les complétives par que, l'alternance entre ces complétives et les complétives réduites à l'infinitif et le choix de la préposition introductrice de l'infinitif (de, $\grave{a}, \varnothing)$. Les apprenants hellénophones, comme les autres apprenants du FLE, achoppent sur ces difficultés, mais sont confrontés en outre à d'autres problèmes dont l'une des sources est leur L1. En effet le grec n'a pas d'infinitif et la nature et les usages de la forme dite 'subjonctif' diffèrent significativement de ceux du français. Nous rendons compte dans cet article de la troisième étape d'un projet concernant l'apprentissage/acquisition et l'enseignement de cette zone de la grammaire du français. Un test de jugement de grammaticalité a été administré à deux groupes d'étudiants (première année et avancés). Sur la base des résultats obtenus, nous montrons quelles structures sont problématiques et candidates à la fossilisation, nous discutons des raisons possibles/probables des difficultés rencontrées par les apprenants dans leurs jugements et nous concluons en suggérant des pistes pour un enseignement plus efficace des complétives aux hellénophones.
\end{abstract}

\footnotetext{
*Corresponding author : frynidoa@ucy.ac.cy
} 


\begin{abstract}
Complement clauses and Greek-speaking learners of French. In grammars and textbooks of French as a Foreign Language, the sections devoted to complement clauses (other than interrogatives) tend to stress three aspects of French grammar: the use of mood (indicative vs subjunctive) in finite complement clauses introduced by $q u e$, the alternation between these finite complement clauses and reduced complement clauses in the infinitive, and the choice of the introductory preposition for the infinitive $(d e, \grave{a}, \varnothing)$. Greek-speaking learners, like other learners of French as a Foreign Language, stumble over these details, but also face other problems, some having their source in their L1. Greek has no infinitive and the nature and uses of the so-called 'subjunctive' form in Greek differ significantly from those of the French subjunctive. In this article we report on the third stage of a project concerning the learning/acquisition and teaching of this area of French grammar. A grammaticality judgement test was administered to two groups of students (first year and advanced). On the basis of the results obtained, we show which structures are problematic and are candidates for fossilization, we discuss the possible/probable reasons for the difficulties encountered by the learners in their judgments and we conclude by suggesting ways of more effectively teaching the syntax of complementation to Greek-speaking learners.
\end{abstract}

\title{
1 Introduction
}

Dans les grammaires et manuels du Français Langue Étrangère (FLE), les chapitres ou sections consacrés aux subordonnées complétives (autres qu'interrogatives) mettent particulièrement l'accent sur trois aspects de ce secteur de la grammaire française : l'emploi du mode (indicatif ou subjonctif) dans les complétives introduites par que, l'alternance entre ces complétives et les complétives réduites à l'infinitif et le choix de la préposition qui gouverne l'infinitif (de, $\grave{a}, \varnothing)$. Les apprenants hellénophones, comme les autres apprenants du FLE, achoppent sur ces difficultés, mais sont confrontés en outre à d'autres problèmes qui compliquent l'apprentissage. L'une des sources de ces problèmes est la langue première (L1). En effet le grec moderne est une langue sans infinitif et la nature et les usages de la forme dite 'subjonctif' ( français. 
Depuis un an nous sommes engagées dans un projet concernant l'apprentissage/acquisition de la complémentation en FLE par des élèves et étudiants hellénophones chypriotes et l'enseignement qui leur est prodigué dans ce domaine. Dans une première étape nous avons constitué un corpus qui nous a permis de cataloguer les erreurs rencontrées dans des travaux écrits d'apprenants et de juger de leur fréquence. La seconde étape a permis, au cours d'un atelier de formation didactique et d'entrevues individuelles avec des professeurs de FLE, de faire des hypothèses sur les origines possibles des erreurs et sur les moyens d'y remédier (voir Monville-Burston \& Kakoyianni-Doa 2017). La troisième phase, celle qui fait l'objet du présent article, a pour but d'essayer de mieux comprendre l'interlangue des apprenants en accédant à leur connaissance internalisée de la question de grammaire investiguée. L'instrument utilisé à cet effet est un test de jugement de grammaticalité (TJG). L'emploi de ce type de test a l'avantage d'ouvrir une fenêtre sur la compétence des apprenants, les données écrites libres ayant déjà fourni des renseignements sur leur performance, sur leur utilisation effective de la langue. On a parfois critiqué la validité des TJG, car divers facteurs interagissent avec les jugements; aussi le projet comportera-t-il une quatrième phase, présentement en progrès : un test de traduction (grecfrançais) complété par des entrevues individuelles au cours desquelles les participants, au-delà de leurs intuitions, expliciteront leur utilisation de telle ou telle structure.

L'article comportera cinq parties : a) Pour contextualiser la partie centrale du développement (le TJG), nous décrirons quelques traits essentiels de la syntaxe de la complémentation en grec et mentionnerons les principaux types d'erreurs rencontrés dans le corpus écrit. b) Puis suivra une présentation du TJG et de la méthodologie utilisée pour l'élaborer et l'administrer et la formulation de nos questions de recherche. c) Nous ferons ensuite état des résultats et d) nous les interpréterons et les discuterons en répondant à nos questions de recherche et en nous focalisant sur les structures qui se seront révélées problématiques et candidates à la fossilisation et sur les raisons possibles/probables des difficultés rencontrées par les apprenants dans leurs jugements. e) Nous conclurons sur des considérations concernant un enseignement de la complémentation qui serait mieux adapté à un public hellénophone. 


\section{Contexte de l'étude}

\subsection{Quelques traits de la syntaxe des complétives en grec}

En grec les complétives se divisent en deux catégories (Mackridge 1985, Holton, Mackridge \& Philippaki-Warburton 1997, Klairis \& Babiniotis 2010) : celles dont le mode est l'indicatif et celles dont le mode est le subjonctif.

Les complétives à l'indicatif sélectionnent une conjonction introductrice qui dépend de la sémantique du verbe qui les gouvernent : par exemple óti/pos (ó $\tau / \pi \omega \varsigma)$ pour les verbes de verbes de parole, de pensée ou de croyance, pou ( $\pi \mathrm{ov})$ pour les verbes factifs, miv/mipos $(\mu \eta v / \mu \eta \dot{\pi} \omega \varsigma)$ pour les verbes de crainte, etc. Toutes ces conjonctions ont pour équivalent français la conjonction que.
(1) Nomizo [óti/pos mou les
psemata]
penser-PRES.1SG CONJ PPERS dire-PRES.IND.2SG des mensonges-ACC

[Je pense que tu me dis des mensonges]

Les complétives au subjonctif ( mot $n a(v \alpha)$ qui précède le verbe subordonné et ne peut en être séparé que par des clitiques. Elles dépendent des verbes de volonté, de désir, de souhait, de demande, d'ordre, etc. et de bon nombre d'expressions impersonnelles. Le statut de $n a$ est controversé. Les grammaires ordinaires et les dictionnaires du grec moderne semblent toutes considérer ce mot comme une conjonction ( $\sigma 0 ́ v \delta \varepsilon \sigma \mu \mathrm{s})$, tandis que chez les linguistes deux camps s'opposent : le camp de na-conjonction et le camp de na-particule modale (morphème inflexionnel marqueur de modalité) (Christidis 1986 ; Philippaki-Warburton 1994).

(2) Thelo [o Pavlos na erthi] vouloir-PRES.1SG le Paul-NOM. PARTIC. venir-SUBJ-3SG Je veux [que Paul vienne].

(3) Prepi [na vroume ena dikigoro] falloir-PRES.3SG PARTIC. trouver-SUBJ-1PL un avocat-ACC. Il faut [que nous trouvions un avocat].

(4) $\mathrm{o}$ theli $[$ na pai mazi tou i Maria

$\begin{array}{llllll}\text { Pavlos } & & & & \\ \text { le Paul- } & \text { vouloir- } & \text { PARTIC. aller-SUBJ-3SG } & \text { avec } \\ \text { NOM PPERS } & \text { PRES.3S } & & & \text { la Maria- }\end{array}$

[Remarquez le rejet du sujet de la complétive en fin de proposition]

Pavlos veut [que Marie aille avec lui]

(5) thelo [psemata na mi mou les]

vouloir- Des mensonges- PARTIC. NEG. PPERS dire-SUBJ-

PRES.1SG ACC 2SG

[Remarquez la place du COD avant la particule modale et les clitiques avant la forme verbale]

Je ne veux pas [que tu me dises des mensonges].

Il est à noter que le grec moderne n'ayant pas d'infinitif, les complétives réduites à l'infinitif comme dans je dois partir, il craint de la rencontrer, etc. sont inexistantes. (6) et

(7) sont au 'subjonctif', et le verbe est fini.

(6) Boro [na figo]

pouvoir-PRES.1SG PARTIC. partir-SUBJ-1SG

Je peux [partir]

(7) Bori [na $\quad$ figi]

pouvoir-PRES.3SG PARTIC. partir-SUBJ-3SG

Il peut [partir] 
Ce sont les difficultés des apprenants liées aux équivalents français de ces complétives grecques au subjonctif qui nous intéressent dans cet article.

\subsection{Des erreurs typiques}

Sur la base de notre corpus nous avons classifié les erreurs les plus fréquentes comme suit. Les difficultés observées concernent typiquement :

a) le lien entre le verbe principal et la complétive (que vs de vs Ø). Ex :

C'est une nécessité *qu' [= d'] apprendre une langue étrangère.

b) l'absence du marqueur de l'infinitif de ou l'absence d'une préposition, dans les complétives réduites. Ex :

Cette association essaye *accueillir [= d'accueillir] des personnes malades.

c) le choix entre la complétive en que et la complétive réduite à l'infinitif. Ex :

Je souhaite *Paphos de gagner [= que Paphos gagne] de nombreux titres.

d) la construction des verbes modaux : pouvoir, vouloir, devoir. Ex :

On peut $*$ de communiquer [= communiquer].

e) la construction des verbes impersonnels. Ex :

Paphos *il faut [= doit] recevoir quelques conseils.

f) la forme du verbe de la complétive en que (temps, mode, etc.). Ex :

Il est inquiétant que les enfants de moins de 15 ans $n$ '*ont [= aient] jamais connu un monde sans Internet.

\section{Méthodologie de l'étude}

\subsection{Les participants}

Vingt-deux étudiants du Département d'Études françaises et Européennes de l'Université de Chypre ayant approximativement le niveau A2-B2 du CECR ont participé à l'étude. Les participants ont tous le grec moderne comme L1 et ont appris l'anglais (L2) avant le français. Le français est une L3 obligatoire dans plus de la moitié de la scolarité des jeunes chypriotes grecs. Parmi les participants les filles (17 étudiantes sur 22) sont surreprésentées par rapport aux garçons (5 étudiants sur 22), mais cela ne semble pas avoir affecté les résultats. Les participants sont inscrits soit dans la filière "Études françaises » ou dans la filière «Études européennes » avec le français comme langue majeure. Pour notre étude, ils ont été répartis en deux groupes expérimentaux : le Groupe 1, constitué d'étudiants de première année (12 étudiants sur 22) et le Groupe 2 (10 étudiants sur 22) constitué d'étudiants de seconde, troisième et quatrième années. Le Groupe 1 est arrivé à l'université avec comme bagage les connaissances du FLE acquises au lycée. Le Groupe 2 a reçu en plus un enseignement universitaire stratifié, c'est-à-dire que la taille du département étant modeste, l'étudiant après la première année suit un ensemble de cours de langue qui peut rassembler des étudiants des trois années supérieures. Il est à noter que le diplôme de finalité est obtenu après quatre ans d'études.

L'échantillon des individus qui ont participé au test est donc un échantillon dit parfois « de convenance », ou encore " groupe intact» (voir par exemple Johnson \& Johnson 1998, Mackay \& Gass 2005, Farrokhi \& Mahmoudi-Hamidabad 2012, Buchstaller \& Khattab 2013). Ce genre d'échantillonnage, basé sur une sélection non-aléatoire, n'est pas représentatif d'une population plus large (inexistante dans le cas considéré ici) et les résultats obtenus ne sont forcément pas généralisables: comme le seul établissement universitaire chypriote qui offre un programme d'études françaises est celui de l'université de Chypre, l'échantillon choisi était le seul qui fût disponible. Il va de soi qu'il faut garder à l'esprit les désavantages de l'échantillonnage de convenance : possibilité de biais, présence 
éventuelle de valeurs aberrantes dans l'échantillon qui menacent l'homogénéité du groupe et faussent les résultats (Farrokhi \& Mahmoudi-Hamidabad 2012), et que cela incite à la prudence au moment de tirer des conclusions. Cependant, bien que la randomisation et la représentativité maximale soient généralement vues comme l'idéal dans les études quantitatives, les circonstances peuvent forcer une démarche différente si la nécessité l'impose comme dans notre cas -démarche qui de plus est justifiable si, comme nous l'avons fait plus haut, on définit avec précision les circonstances dans lesquelles la recherche a été conduite (Farrokhi \& Mahmoudi-Hamidabad 2012). Buchstaller \& Khattab (2013) notent qu'il est impossible de procéder autrement quand par exemple les langues en voie de disparition sont l'objet de la recherche, et ajouterons-nous, dans le contexte de langues "modimes» (Langues Moins Diffusées et Moins Enseignées) où une langue étrangère (ici le FLE, langue tierce) est enseignée à des apprenants d'une langue modime (la variété chypriote du grec). D'autre part, comme le font remarquer Johnson et Johnson (1998), même si une recherche basée sur un échantillonnage de convenance n'est pas la plus appropriée selon les modèles statistiques classiques, il n'en demeure pas moins que les résultats qu'elle produit permettent de mettre en lumière des faits ou des tendances qui sont révélateurs.

\subsection{Le TJG : son élaboration, son déroulement}

Le TJG contient 20 items, 9 corrects et 11 incorrects [voir (8)].

(8) Phrases à juger

1. Il faut que les choses changent.

2. *Twitter peut devient une addiction.

3. Il est indispensable que notre capitale se modernise.

4. *Je pense que c'est important donnons le bon exemple.

5. *Je voudrais d'aller faire du ski pendant les vacances.

6. Il est très important de savoir nager.

7. Mon père ne veut jamais avoir tort.

8. *Il faut votre exercice de donner avant lundi prochain.

9. Il est nécessaire d'avoir un passeport pour aller en Australie.

10. *Je souhaite mon frère de se marier avec ma meilleure copine.

11. *Chaque citoyen peut différentes choses de faire pour sauver l'environnement.

12. La directrice voudrait que tous les enfants soient à l'école à $7 \mathrm{~h} 30$.

13. Tu devrais payer tout de suite ton loyer.

14. Les parents doivent surveiller ce que leurs enfants regardent à la télévision.

15. *Les autorités il faut assurer la sécurité des réfugiés.

16. *Nous pouvons d'écouter de la musique, si tu veux.

17. *Nous sommes en retard. Il faut partir tout de suite et de ne pas attendre Maria.

18. *Il est nécessaire d'il y a une bonne organisation dans une entreprise.

19. Pour votre santé il faut faire du sport.

20. *De nos jours l'utilisation de l'ordinateur est très importante de maitriser.

Le TJG a été passé pendant un cours d'expression écrite. Les items à juger ont été lus à haute voix par l'administrateur du test avec une pause de 20 secondes entre chaque lecture. Pendant cette pause les sujets avaient à décider si la phrase proposée était selon eux (1) correcte, (2) probablement correcte, (3) probablement incorrecte ou (4) incorrecte et, dans le dernier cas, pour rectifier la phrase, ce qu'ils ont fait très souvent, parfois en commettant une autre erreur. Le TJG a été configuré en tenant compte des remarques méthodologiques de spécialistes de ce type d'évaluation comme, entre autres, Chaudron (1983), Ellis (1991), Cowan \& Hatasa (1994), Tremblay (2005) (voir aussi Monville- 
Burston 2008). Nous sommes conscientes des critiques qui ont été faites à ce genre d'instrument : par exemple les participants devinent et décident au hasard s'ils se sentent incapables de répondre. La gamme de quatre choix a permis cependant aux sujets de ne pas porter un jugement catégorique s'ils hésitaient. Pour limiter la variabilité des jugements nous avons utilisé dans les items un vocabulaire simple, connu des sujets, et nous avons créé des phrases courtes afin qu'ils puissent se concentrer rapidement sur la grammaticalité ou la non-grammaticalité de la structure proposée. Les structures sur lesquelles les participants ont été invités à porter un jugement ont été déterminées par les types d'erreurs récurrentes décrites en 2.2. Cependant à la suite de la seconde phase du projet nous avons décidé de donner un poids particulier a) aux structures qui sont des calques des structures grecques, b) à la construction des verbes modaux (pouvoir, vouloir, devoir) et des expressions impersonnelles exprimant l'obligation ou le souhait et c) au terme introducteur de la complétive (que, de). Les phrases choisies ont été tirées ou adaptées de phrases du corpus écrit ou modelées sur des exemples qu'on y a relevés. Pour l'analyse des résultats, quelques adaptations ont été nécessaires. En effet comme les jugements concernaient des phrases entières et non pas une forme grammaticale isolée, certains items ont été jugés incorrects pour des raisons qui n'avaient rien à voir avec la complémentation, comme l'ont montré certaines corrections proposées quand un item était jugé incorrect. Par exemple dans la phrase 16 Nous pouvons d'écouter de la musique, si tu veux, un étudiant a changé l'article partitif en une préposition et écrit Nous pouvons d'écouter à la musique, si tu veux.

Sur la base des observations faites et des éclairages acquis au cours des étapes 1 et $2 \mathrm{du}$ notre projet, nous formulons nos questions de recherche comme suit :

a) Quelles structures entraînent le plus/le moins de jugements déviants ?

b) Le verbe (ou l'expression verbale) introducteur de la complétive a-t-il une incidence sur le jugement des sujets?

c) Pour quelles structures l'influence de la L1 est-elle la plus sensible dans les jugements des sujets?

d) La comparaison entre les jugements du Groupe 1 et du Groupe 2 montre-t-elle un progrès? Confirme-t-elle les remarques des enseignants que certains aspects de la complémentation sont des zones de fossilisation potentielle?

\subsection{Procédure d'analyse des données du TJG}

Pour l'établissement des résultats, nous avons procédé de la façon suivante: dans un premier temps, nous avons recensé les différents types de réponses (correct, probablement correct, probablement incorrect, incorrect) des apprenants de chaque groupe (Groupe 1, $\mathrm{n}=12$; Groupe $2, \mathrm{n}=10$ ) pour chaque question. Notons que deux apprenants ( $\mathrm{A} 3$ et $\mathrm{C} 1$ ) n'ont pas répondu à deux questions (cf. question 13 et 12 respectivement) : le nombre des réponses traitées pour ces questions est donc inférieur par rapport aux autres réponses $(\mathrm{n}=21$, au lieu de $\mathrm{n}=22)$. Dans un deuxième temps, nous avons effectué certaines adaptations quand la correction proposée pour «incorrect» était inappropriée. En effet l'examen des réponses a montré que cinq participants (A6, A7, A11, B4, D1) ont jugé deux items 16 et 20 comme incorrects et ont corrigé dans la phrase un point qui était correct (voir exemple en 3.2). Nous avons alors changé la réponse donnée «incorrect» en «probablement correct». Dans un troisième temps, pour faciliter l'analyse des données, qui se sont révélées complexes, et parce que dans l'ensemble les participants ont préféré les options extrêmes «correct et incorrect» (cf. 4 infra) nous avons fait passer les réponses " probablement correct» dans la catégorie « correct» et « probablement incorrect» dans la catégorie " incorrect». Enfin, nous avons calculé nos résultats en pourcentage et réorganisé tous les items selon la qualité des jugements faits par les sujets, de l'item le mieux jugé à l'item le moins bien jugé. Nous avons aussi calculé les pourcentages d'amélioration des jugements entre le Groupe 1 et le Groupe 2. 


\section{Résultats}

Les résultats généraux sont consignés dans la Figure 1. Dans l'ensemble les participants ont choisi les options "Correct/Incorrect» (jugements catégoriques) plutôt que les jugements intermédiaires («Plutôt correct/Plutôt incorrect»). Certains items cependant recueillent un nombre supérieur de ces derniers ou un nombre égal des deux types et témoignent donc d'une incertitude sur la grammaticalité. Il s'agit des phrases 6, 7, 13, 17, 18.

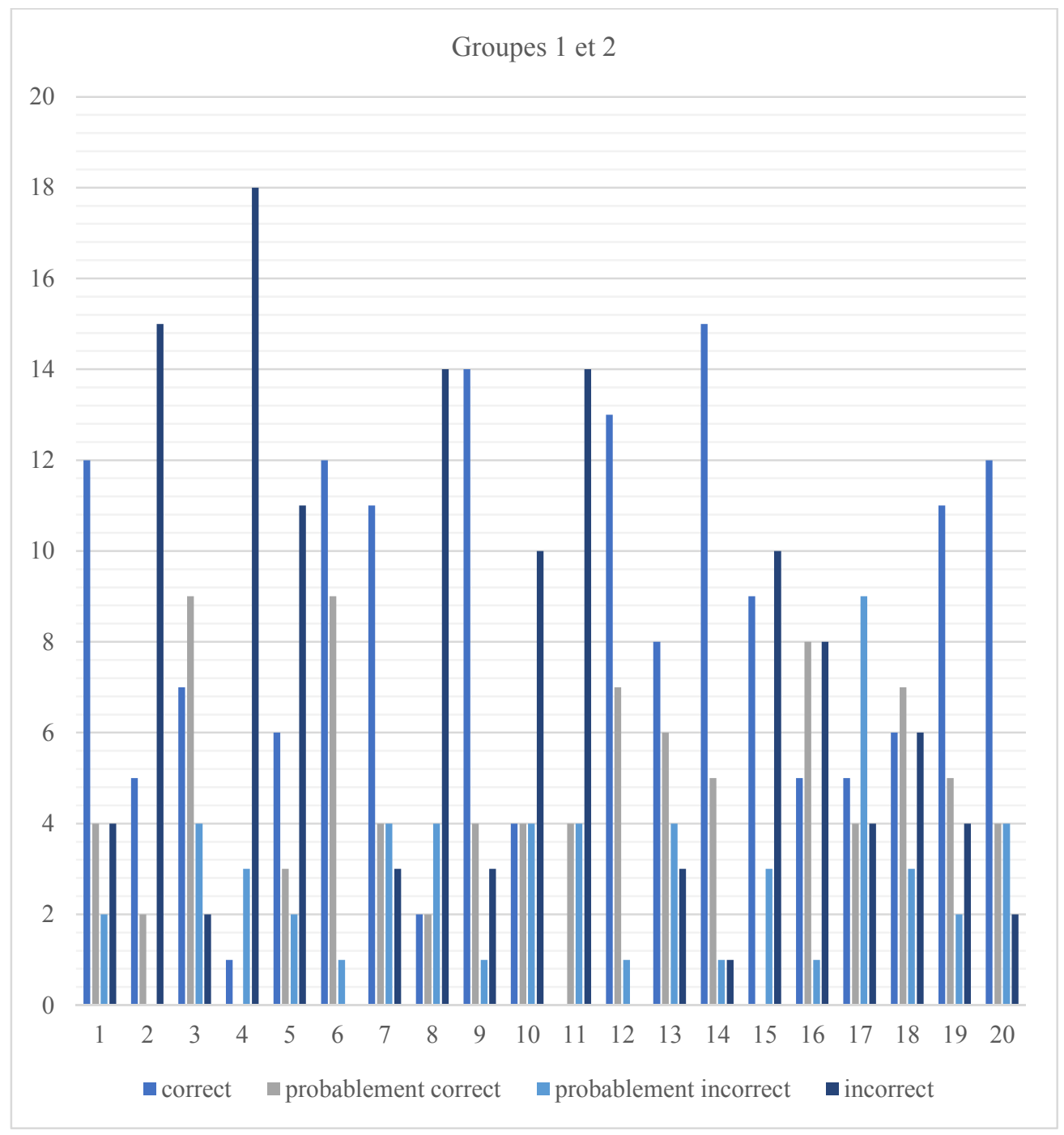

Fig. 1. Résultats généraux. 
Pour dix-sept des items sur vingt les bons jugements dominent, mais avec des proportions variées par rapport aux mauvais jugements (Voir Tableau 1). Les mauvais jugements culminent avec l'item 20 estimé correct par 16 étudiants sur 22. Les items les mieux jugés $(4,6,12$ et 14) l'ont été par 20-21 étudiants sur 22. L'éventail de qualité des jugements pour l'ensemble des sujets (aussi bien que pour les sujets de chaque groupe) est donc étendu.

Tableau 1. Qualité des jugements

\begin{tabular}{|c|c|c|c|c|c|c|}
\hline & \multicolumn{3}{|c|}{ Bon jugement } & \multicolumn{3}{c|}{ Mauvais jugement } \\
\hline & Tous & Groupe 1 & Groupe 2 & Tous & Groupe 1 & Groupe 2 \\
\hline$(1)$ & $16 / 22$ & $10 / 12$ & $6 / 10$ & $6 / 22$ & $2 / 12$ & $4 / 10$ \\
\hline$(2)$ & $15 / 22$ & $8 / 12$ & $7 / 10$ & $7 / 22$ & $4 / 12$ & $3 / 10$ \\
\hline$(3)$ & $16 / 22$ & $8 / 12$ & $8 / 10$ & $6 / 22$ & $4 / 12$ & $2 / 10$ \\
\hline$(4)$ & $21 / 22$ & $11 / 12$ & $10 / 10$ & $1 / 22$ & $1 / 12$ & $0 / 10$ \\
\hline$(5)$ & $13 / 22$ & $7 / 12$ & $6 / 10$ & $9 / 22$ & $5 / 12$ & $4 / 10$ \\
\hline$(6)$ & $21 / 22$ & $11 / 12$ & $10 / 10$ & $1 / 22$ & $1 / 12$ & $0 / 10$ \\
\hline$(7)$ & $15 / 22$ & $9 / 12$ & $6 / 10$ & $7 / 22$ & $3 / 12$ & $4 / 10$ \\
\hline$(8)$ & $18 / 22$ & $9 / 12$ & $9 / 10$ & $4 / 22$ & $3 / 12$ & $1 / 10$ \\
\hline$(9)$ & $18 / 22$ & $9 / 12$ & $9 / 10$ & $4 / 22$ & $3 / 12$ & $1 / 10$ \\
\hline$(10)$ & $14 / 22$ & $10 / 12$ & $6 / 10$ & $8 / 22$ & $2 / 12$ & $4 / 10$ \\
\hline$(11)$ & $18 / 22$ & $8 / 12$ & $10 / 10$ & $4 / 22$ & $4 / 12$ & $0 / 10$ \\
\hline$(12)$ & $20 / 21$ & $12 / 12$ & $8 / 9$ & $1 / 22$ & $0 / 12$ & $1 / 9$ \\
\hline$(13)$ & $14 / 21$ & $7 / 11$ & $7 / 10$ & $7 / 22$ & $4 / 11$ & $3 / 10$ \\
\hline$(14)$ & $20 / 22$ & $10 / 12$ & $10 / 10$ & $2 / 22$ & $2 / 12$ & $0 / 10$ \\
\hline$(15)$ & $12 / 21$ & $8 / 12$ & $4 / 9$ & $9 / 22$ & $4 / 12$ & $5 / 9$ \\
\hline$(16)$ & $9 / 22$ & $5 / 12$ & $4 / 10$ & $13 / 22$ & $7 / 12$ & $6 / 10$ \\
\hline$(17)$ & $13 / 22$ & $7 / 12$ & $6 / 10$ & $9 / 22$ & $5 / 12$ & $4 / 10$ \\
\hline$(18)$ & $9 / 22$ & $5 / 12$ & $4 / 10$ & $13 / 22$ & $7 / 12$ & $6 / 10$ \\
\hline$(19)$ & $16 / 22$ & $7 / 12$ & $9 / 10$ & $6 / 22$ & $5 / 12$ & $1 / 10$ \\
\hline$(20)$ & $6 / 22$ & $3 / 12$ & $3 / 10$ & $16 / 22$ & $9 / 12$ & $7 / 10$ \\
\hline
\end{tabular}

\section{Discussion}

\subsection{Qualité du jugement des participants}

Le développement qui suit nous permettra de répondre aux questions de recherche a), b) et c). Nous nous concentrons sur les jugements les plus extrêmes. Nous considérons d'abord les items où le jugement de l'ensemble des participants a été contraire à la grammaire du français dans au moins $50 \%$ des cas. Il s'agit de :

16. *Nous pouvons d'écouter de la musique, si tu veux.

18. *Il est nécessaire d'il y a une bonne organisation dans une entreprise.

20. *De nos jours l'utilisation de l'ordinateur est très importante de maîtriser.

Pour 16, vient immédiatement à l'esprit l'hypothèse que la structure de la L1 peut avoir occasionné les mauvais jugements. L'acceptation de l'item résulte probablement du

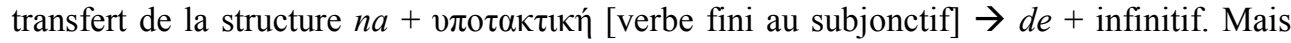
cette explication est probablement insuffisante car le mauvais jugement peut, de façon cumulative, venir aussi d'une généralisation des structures complétives infinitives en de, les plus communes en français. On suppose donc que les apprenants ont dans leur interlangue 
une règle [Règle $\mathrm{A}]$ qui se formulerait ainsi : $n a+$ verbe fini $=d e+$ infinitif, où la présence d'un terme introducteur est vue comme essentielle. La règle n'est pas sans validité : elle dit que l'équivalent de $n a$ est de, marqueur d'infinitif (cf. Kalmbach 2008 et aussi Sandfeld 1965, Riegel et al. 2016). On trouve en outre une règle similaire en anglais, la L2 des participants (I want to go, I need to go, etc.). La Règle A est appliquée dans les phrases 6 et 9 par exemple, qui sont parmi les mieux jugées du test (voir plus bas). Cependant elle souffre des exceptions. On doit en exclure les verbes introducteurs modaux vouloir, pouvoir, devoir, falloir, d'où l'item agrammatical 5 *Je voudrais d'aller faire du ski pendant les vacances, pourtant jugé correct par 9 étudiants sur 22. Inversement les items 7 Mon père ne veut jamais avoir tort, $13 \mathrm{Tu}$ devrais payer tout de suite ton loyer et 19 Pour votre santé il faut faire du sport, bien que grammaticaux ont été jugés incorrects par7 étudiants sur 22. Pour à peu près un tiers de l'ensemble des participants, l'exception à la Règle A n'est donc pas véritablement assimilée.

Le cas de la phrase 18 peut être discuté en liaison avec les phrases 2 et 4 . Pour les participants qui acceptent $2 *$ Twitter peut devient une addiction et $4{ }^{*} J e$ pense que c'est important donnons le bon exemple (qui contiennent des erreurs souvent rencontrées en

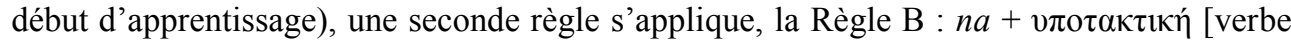
fini au subjonctif] $=\varnothing+$ verbe fini. Ce qui est retenu pour essentiel par l'apprenant, c'est la forme verbale finie du grec à laquelle il fait correspondre une forme verbale finie en français, sans marqueur. La présence du marqueur de, jugée correcte, est intrigante dans 18. On peut se demander si l'association de de et d'il y a n'est pas provoquée par une incertitude sur la forme d'infinitif à donner à il y $a$. Les jugements sur 18 montrent que des hésitations demeurent encore sur le terme introducteur des complétives.

Pour comprendre les jugements défectueux sur l'item 20, il est indispensable de faire appel aux structures de la L1. Il est intéressant d'associer à la discussion de 20, pour le Groupe 2, les items 10 *Je souhaite mon frère de se marier avec ma meilleure copine et 15 * Les autorités il faut assurer la sécurité des réfugiés, deux phrases qui ont été mieux jugées par le Groupe 1. Ci-dessous nous mettons en parallèle (dans (9), (10) et (11)) l'item du test, la phrase grecque correspondante et une analyse syntaxique/pragmatique de cette phrase.

(9) (Item 20) *l'utilisation de l'ordinateur est très importante de maîtriser.

[Tin chrisi tou ipologisti], ine poli simantiko na gnorizi kanis

THÈME=l'utilisation de l'ordinateur [Groupe nominal-accusatif]; RHÈME=être [3SG-PRES-Impers.] + très important [Adj. Attrib-nominatif neutre] + connaître $[n a+3 \mathrm{SG}-\mathrm{SUBJ}]+$ quelqu'un [Pronom-Nominatif]

(10) (Item 10)*Je souhaite mon frère de se marier avec ma meilleure copine.

Efchome [o adelfos mou] na pantrefti me tin kaliteri mou fili

Je souhaite THÈME=mon frère [Groupe nominal-nominatif]; RHÈME=se marier [na + 3SG-SUBJ] + avec ma meilleure copine [Groupe prépositionnel].

(11) (Item 15)* Les autorités il faut assurer la sécurité des réfugiés.

[I arches] prepi na diasfalizoun tin asfalia ton prosfigon

THÈME=Les autorités [Groupe nominal-nominatif]; RHÈME=il faut [3SG-PRES Impers.] + assurer [na + 3PL-SUBJ] + la sécurité des réfugiés [Groupe nominalaccusatif]

Ce qui cause problème aux sujets ici, c'est l'ordre des mots du grec qu'ils reproduisent en français. Dans l'item 20 l'objet du verbe de la complétive est projeté en début d'énoncé par une dislocation à gauche ; dans l'item 15 c'est le sujet de la complétive qui est placé en tête de phrase avant le verbe principal et la complétive. Un autre type de transfert de l'ordre des mots est représenté dans l'item 10 où le sujet de la complétive est placé en tête de la subordonnée avant le groupe verbal. Ces structures sont dues à l'ordre flexible des mots dans la phrase grecque où des éléments phrastiques peuvent être thématisés/rhématisés de façons variées. ${ }^{i}$ Il est à noter d'autre part que le verbe grec impersonnel prepi a pour équivalents français il faut (verbe impersonnel) aussi bien que devoir (verbe personnel) et 
que cette situation engendre souvent des confusions dans l'utilisation des deux verbes français. Falloir est en quelque sorte rendu personnel comme dans l'item 15. Par ailleurs, dans la phrase 20 on peut supposer que l'influence de l'anglais favorise aussi l'acceptabilité de l'agrammaticalité de la structure (... is important to master).

Les items les mieux jugés par l'ensemble des participants (au moins 16 étudiants sur 22) sont :

1. Il faut que les choses changent.

3. Il est indispensable que notre capitale se modernise.

4. *Je pense que c'est important donnons le bon exemple.

6 . Il est très important de savoir nager.

8. *Il faut votre exercice de donner avant lundi prochain.

9. Il est nécessaire d'avoir un passeport pour aller en Australie.

11. *Chaque citoyen peut différentes choses de faire pour sauver l'environnement.

12. La directrice voudrait que tous les enfants soient à l'école à $7 \mathrm{~h} 30$.

14. Les parents doivent surveiller ce que leurs enfants regardent à la télévision.

19. Pour votre santé il faut faire du sport.

On remarque que -si leur agrammaticalité est corrigée- sept de ces phrases contiennent des complétives qui dépendent d'expressions impersonnelles (il est important, il faut, il est nécessaire, il est indispensable) et que pour cinq d'entre elles les complétives qui y sont enchâssées sont des subordonnées non réduites en que avec un verbe fini. Faut-il supposer que ces facteurs sont favorables à l'appropriation des complétives par les apprenants hellénophones ? On note aussi que la construction de il faut est bien jugée comme correcte $(1,19)$ ou fautive (8) si l'impersonnel vient en tête de phrase et que l'ordre des mots standard n'est pas perturbé pour des raisons de thématisation (comparer avec la phrase 15 discutée plus haut). Au final il apparaît que généralement dans notre test, ce sont les verbes modaux non-impersonnels (vouloir, pouvoir, devoir) suivis de complétives réduites à l'infinitif qui provoquent le plus de flottements dans la reconnaissance de la grammaticalité des phrases proposées aux participants, ainsi que les phrases erronées qui reproduisent l'ordre des mots du grec.

\subsection{Entre Groupe 1 et Groupe 2 : Amélioration des jugements}

Pour répondre à la quatrième question de recherche, nous comparons la qualité des jugements des participants du Groupe 1 et du Groupe 2 afin d'établir s'il y a eu amélioration ou non et pour quels aspects de la complémentation. Comme on le voit dans le Tableau 2, les résultats montrent qu'il y a en général des progrès du Groupe le moins avancé au Groupe le plus avancé. Le taux d'amélioration des jugements monte à $33.33 \%$ pour la phrase 11 et à $31,6 \%$ pour la phrase 19 . Pour le reste des phrases le taux d'amélioration est nettement plus bas. Il se situe entre $16,67 \%$ et $1,67 \%$. En ce qui concerne les phrases qui contiennent des formes impersonnelles du type il est nécessaire, il est indispensable, il est important $(9,3,6)$, l'amélioration varie entre $15 \%$, et $8.33 \%$, même si on n'observe pas de progrès pour l'item 18 discuté plus haut. 
Tableau 2. Amélioration des jugements

\begin{tabular}{|c|c|c|c|c|c|}
\hline & \multicolumn{2}{|c|}{ Groupe 1} & \multicolumn{2}{|c|}{ Groupe 2} & \multirow{2}{*}{$\frac{\text { Amélioration }}{\begin{array}{c}\text { Bon } \\
\text { jugement }\end{array}}$} \\
\hline & $\begin{array}{c}\text { Bon } \\
\text { jugement }\end{array}$ & $\begin{array}{c}\text { Mauvais } \\
\text { jugement }\end{array}$ & $\begin{array}{c}\text { Bon } \\
\text { jugement }\end{array}$ & $\begin{array}{l}\text { Mauvais } \\
\text { jugement }\end{array}$ & \\
\hline $\begin{array}{l}\text { (11) *Chaque citoyen peut } \\
\text { différentes choses de faire } \\
\text { pour sauver l'environnement. }\end{array}$ & $8 / 12$ & $4 / 12$ & $10 / 10$ & $0 / 10$ & $33.33 \%$ \\
\hline $\begin{array}{l}\text { (19) Pour votre santé il faut } \\
\text { faire du sport. }\end{array}$ & $7 / 12$ & $5 / 12$ & $9 / 10$ & $1 / 10$ & $31.67 \%$ \\
\hline $\begin{array}{l}\text { Les parents doivent } \\
\text { surveiller ce que leurs enfants } \\
\text { regardent à la télévision. }\end{array}$ & $10 / 12$ & $2 / 12$ & $10 / 10$ & $0 / 10$ & $16.67 \%$ \\
\hline $\begin{array}{l}\text { (8) *Il faut votre exercice de } \\
\text { donner avant lundi prochain. }\end{array}$ & $9 / 12$ & $3 / 12$ & $9 / 10$ & $1 / 10$ & $15.00 \%$ \\
\hline $\begin{array}{l}\text { (9) Il est nécessaire d'avoir } \\
\text { un passeport pour aller en } \\
\text { Australie. }\end{array}$ & $9 / 12$ & $3 / 12$ & $9 / 10$ & $1 / 10$ & $15.00 \%$ \\
\hline $\begin{array}{l}\text { (3) Il est indispensable que } \\
\text { notre capitale se modernise. }\end{array}$ & $8 / 12$ & $4 / 12$ & $8 / 10$ & $2 / 10$ & $13.33 \%$ \\
\hline $\begin{array}{l}\text { (4) *Je pense que c'est } \\
\text { important donnons le bon } \\
\text { exemple. }\end{array}$ & $11 / 12$ & $1 / 12$ & $10 / 10$ & $0 / 10$ & $8.33 \%$ \\
\hline $\begin{array}{l}\text { (6) Il est très important de } \\
\text { savoir nager. }\end{array}$ & $11 / 12$ & $1 / 12$ & $10 / 10$ & $0 / 10$ & $8.33 \%$ \\
\hline $\begin{array}{l}\text { (13) Tu devrais payer tout de } \\
\text { suite ton loyer. }\end{array}$ & $7 / 11$ & $4 / 11$ & $7 / 10$ & $3 / 10$ & $6.36 \%$ \\
\hline $\begin{array}{l}(20) \text { *De nos jours } \\
\text { l'utilisation de l'ordinateur } \\
\text { est très importante de } \\
\text { maîtriser. }\end{array}$ & $3 / 12$ & $9 / 12$ & $3 / 10$ & $7 / 10$ & $5.00 \%$ \\
\hline $\begin{array}{l}\text { (2) *Twitter peut devient une } \\
\text { addiction. }\end{array}$ & $8 / 12$ & $4 / 12$ & $7 / 10$ & $3 / 10$ & $3.33 \%$ \\
\hline $\begin{array}{l}\text { (5) *Je voudrais d'aller faire } \\
\text { du ski pendant les vacances. }\end{array}$ & $7 / 12$ & $5 / 12$ & $6 / 10$ & $4 / 10$ & $1.67 \%$ \\
\hline $\begin{array}{l}\text { (17) *Nous sommes en } \\
\text { retard. Il faut partir tout de } \\
\text { suite et de ne pas attendre } \\
\text { Maria. }\end{array}$ & $7 / 12$ & $5 / 12$ & $6 / 10$ & $4 / 10$ & $1.67 \%$ \\
\hline $\begin{array}{l}(16) * \text { Nous pouvons } \\
\text { d'écouter de la musique, si tu } \\
\text { veux. }\end{array}$ & $5 / 12$ & $7 / 12$ & $4 / 10$ & $6 / 10$ & $-1.67 \%$ \\
\hline $\begin{array}{l}(18) \text { *Il est nécessaire d'il y a } \\
\text { une bonne organisation dans } \\
\text { une entreprise. }\end{array}$ & $5 / 12$ & $7 / 12$ & $4 / 10$ & $6 / 10$ & $-1.67 \%$ \\
\hline $\begin{array}{l}\text { (12) La directrice voudrait } \\
\text { que tous les enfants soient à } \\
\text { l'école à } 7 \mathrm{~h} 30 \text {. }\end{array}$ & $12 / 12$ & $0 / 12$ & $8 / 9$ & $1 / 9$ & $-11.11 \%$ \\
\hline $\begin{array}{l}\text { (7) Mon père ne veut jamais } \\
\text { avoir tort. }\end{array}$ & $9 / 12$ & $3 / 12$ & $6 / 10$ & $4 / 10$ & $-15.00 \%$ \\
\hline $\begin{array}{l}\text { (15) *Les autorités il } \\
\text { assurer la faut } \\
\text { réfugiés. }\end{array}$ & $8 / 12$ & $4 / 12$ & $4 / 9$ & $5 / 9$ & $-22.22 \%$ \\
\hline $\begin{array}{l}\text { (1) Il faut que les choses } \\
\text { changent. }\end{array}$ & $10 / 12$ & $2 / 12$ & $6 / 10$ & $4 / 10$ & $-23.33 \%$ \\
\hline
\end{tabular}




\begin{tabular}{|l|c|c|c|c|c|}
\hline & \multicolumn{2}{|c|}{ Groupe 1 } & \multicolumn{2}{c|}{ Groupe 2 } & Amélioration \\
\hline & $\begin{array}{c}\text { Bon } \\
\text { jugement }\end{array}$ & $\begin{array}{c}\text { Mauvais } \\
\text { jugement }\end{array}$ & $\begin{array}{c}\text { Bon } \\
\text { jugement }\end{array}$ & $\begin{array}{c}\text { Mauvais } \\
\text { jugement }\end{array}$ & $\begin{array}{c}\text { Bon } \\
\text { jugement }\end{array}$ \\
\hline $\begin{array}{l}(10) * \text { Je souhaite mon frère } \\
\text { de se marier avec ma } \\
\text { meilleure copine. }\end{array}$ & $10 / 12$ & $2 / 12$ & $6 / 10$ & $4 / 10$ & $-43.33 \%$ \\
\hline
\end{tabular}

D'autre part pour les phrases $16,18,12,7,15,1$ et 10, nous remarquons une régression, qui est faible dans le cas des deux premières et donc peu significative. Pour les cinq autres elle est plus claire (entre $11 \%$ et $43 \%$ ) et peut paraître étonnante, en particulier pour la phrase 12. Ces phrases contiennent en effet des structures similaires à celles pour lesquelles les jugements ont aussi progressé. Cependant l'identité des structures erronées de 15 et de 10 avec les structures parallèles du grec peut expliquer qu'un fort transfert de la L1 au français entrave les bons jugements. Pour la phrase 7 la négation ne...jamais, où le forclusif est plus sémantiquement chargé, moins fréquent que pas peut aussi avoir eu une influence sur la qualité des jugements.

Au total, on remarque un progrès de la qualité des jugements de moins de $35 \%$ pour 14 des items, mais une régression pour 7 d'entre eux, celle-ci atteignant $44 \%$ dans le cas de l'item 10. Ces résultats semblent confirmer que l'acquisition des points de grammaire considérés dans le TJG est difficile pour les hellénophones et que l'appropriation de ces derniers n'est pas encore fermement établie au niveau universitaire. Ces résultats corroborent aussi les convictions des enseignants (enquêtés dans la phase 2 du projet) que les erreurs sur la syntaxe de la complémentation peuvent facilement se fossiliser, mais en les nuançant. La construction des verbes modaux (pouvoir, vouloir, devoir) paraît être acquise lentement et les risques d'erreur viennent surtout de calques possibles des structures grecques thématisées ; mais les complétives introduites par des expressions impersonnelles semblent être mieux acquises et plus ouvertes vers des progrès.

\section{Conclusion et perspectives didactiques}

Durant la deuxième phase de notre recherche, lors de l'atelier de formation didactique, nous avons pu observer chez certains enseignants beaucoup d'insécurité concernant les structures complétives du français. Face à des phrases fautives du type de celles contenues dans le TJG, ils n'ont pas su corriger certaines des erreurs ou les ont corrigées en en commettant une autre. Nous avions là la preuve que la syntaxe de complémentation du français est un domaine difficile pour les hellénophones et que les enseignants chypriotes ne sont pas toujours dans ce secteur de la grammaire française les meilleurs modèles pour leurs élèves.

Quant aux exercices traditionnels (à trous, à choix multiple, à dominante morphologique, etc.) largement utilisés par le corps enseignant à Chypre, ils s'avèrent n'être pas particulièrement efficaces dans l'acquisition des aspects de la complémentation discutés ici. Il en est de même des explications relatives au fonctionnement de ces phénomènes grammaticaux données en classe. En effet, l'apprenant n'est pas en mesure de rendre opérationnelles les règles de grammaire qu'on lui propose puisque son vécu grammatical s'intercale. Même si les apprenants sont capables d'énoncer des connaissances apprises sous une forme déclarative, ils ne sont pas capables de les appliquer dans la production. Selon Trévise, il s'agirait «d'un fonctionnement à vide de microsystème (...), d'une pensée sans représentation. Il n'y a dans l'esprit que la représentation des signes, mais pas la représentation de ce que ces signes signifient» (1996:34). C'est pour cela que la condition sine qua non de l'efficacité du discours grammatical des enseignants consisterait plutôt à impliquer l'apprenant et à prendre en compte sa culture linguistique. Il s'ensuit que, dans le cas qui nous intéresse ici, les formateurs chypriotes auraient tout intérêt à inclure dans leur programme de stages de formation initiale et continue non seulement une ou plusieurs sessions consacrées à la question de la complémentation, mais 
aussi à faire réfléchir leurs stagiaires sur des façons plus efficaces ou motivantes d'enseigner les structures complétives.

Dans la salle de classe, les enseignants pourraient utiliser des stratégies de remédiation variées, comme certains l'ont suggéré eux-mêmes lors de l'atelier et des entrevues mentionnés plus haut : enseignement en spirale, reprise et répétition, traduction des phrases erronées en L1, incitation à l'autocorrection pour retrouver la règle, etc. Cependant il serait aussi utile d'inviter la réflexion métalinguistique par des tâches réflexives de verbalisation inspirées des exercices de conceptualisation de Besse (1974). L'objectif consisterait à encourager les apprenants à expliquer la façon dont ils construisent leurs représentations sur le fonctionnement des diverses facettes de la complémentation en français. Des activités de jugement de grammaticalité et/ou des corrections sur des phrases erronées produites par les apprenants eux-mêmes pourraient être proposées comme des activités à part entière. Les commentaires des apprenants, leurs incertitudes ou leurs autocorrections issues de l'activité qui est en réalité une vraie tâche, viseraient à développer chez eux la capacité de comprendre les combinaisons entre les différents éléments de la langue et de les appliquer. Comme le déclare Besse, il est possible de contourner une difficulté «en partant, non d'exemples choisis par les grammairiens ou par le professeur, mais des productions des étudiants en L2 » (1984 : 20). Dans cette perspective heuristique, par ces activités de conceptualisation, malheureusement mises de côté depuis l'adoption de l'approche communicative, les apprenants seraient véritablement acteurs de leur apprentissage grammatical (Besse 1984, Besse \& Porcher 1991).

Rappelons que notre étude nous a permis d'identifier certaines marques des représentations sous-jacentes au fonctionnement de la complémentation en français chez les apprenants chypriotes. En l'occurrence, elle a révélé que, pour juger de l'acceptabilité de certaines phrases françaises, les participants se réfèrent souvent à leur savoir grammatical en L1, bien qu'ils aient été exposés à un discours grammatical explicite concernant les éléments qui constituent la complémentation du français. Ils semblent filtrer ce discours à travers le prisme de représentations catégorielles du grec moderne (cf. section 5.1). Dans cette perspective, l'utilisation de la langue maternelle dans la salle de classe pourrait aussi contribuer à la meilleure compréhension des différents phénomènes grammaticaux d'une langue étrangère à condition qu'elle soit accompagnée d'une vraie réflexion métalinguistique. Le fait de comparer les deux systèmes syntaxiques peut permettre à l'apprenant de voir les points communs ou les différences entre sa langue et le français. Comme dit Castellotti, la langue maternelle de l'apprenant « peut devenir un tremplin, dans la mesure où les capacités, notamment d'ordre métalinguistique et métacommunicatif, [...] sont réinjectées dans l'approche d'une nouvelle langue et offrent des outils heuristiques pour entrer dans cette langue et y élargir ses compétences (Castellotti, 2001 : 43-44). On peut penser aussi au programme de l'éveil aux langues élaboré par Candelier, qui cherche à induire chez l'élève le développement d'une ouverture à la diversité linguistique en même temps que des aptitudes métalinguistiques et des capacités d'observation et de raisonnement qui faciliteront la maîtrise des langues à apprendre (voir par exemple Candelier 2003).

Enfin, les grammaires et manuels du FLE utilisés dans les écoles sont le plus souvent des ouvrages publiés en France et qui ne traitent pas les problèmes spécifiques des hellénophones. Il en est de même de la plupart des grammaires/manuels publiés localement, en Grèce et à Chypre, qui tendent à copier les ouvrages publiés en France. Certes certaines grammaires bilingues ou rédigées entièrement en grec s'essaient à accommoder leurs descriptions pour des hellénophones, en donnant par exemple dans leurs chapitres sur la complémentation des traductions dans la L1, en faisant des remarques métalinguistiques ou terminologiques sur la nature de l'infinitif ou du subjonctif ou en accordant une importance marquée à la construction des expressions impersonnelles. Mais rien n'est dit explicitement sur le problème épineux des transferts causés les différences entre l'ordre des mots en grec et en français dans les structures complétives (Monville-Burston \& Kakoyianni-Doa 2017). 
Il est donc nécessaire de mieux «contextualiser » (au sens de Besse 1984, ou encore de Beacco et al. 2014, Fouillet et al. 2015) les descriptions et explications des grammaires pour hellénophones en tenant compte des erreurs récurrentes, des zones potentielles de fossilisation et des connaissances grammaticales antérieures des apprenants sur leur langue maternelle. Ajoutons pour conclure qu'au-delà du projet discuté dans le présent article, un projet plus étendu est en voie de développement pour la production de leçons et d'exercices/activités FLE consacrés tout particulièrement aux difficultés grammaticales rencontrées par les hellénophones, dont celles causées par la syntaxe des complétives.

\section{Références bibliographiques}

Beacco, J.C. Kalmbach, J.-M. \& López, J. S. (dir.). (2014). Les contextualisations de la description du français dans les grammaires étrangères. Langue française, 181.

Besse, H. (1974). Les exercices de conceptualisation ou la réflexion grammaticale au niveau 2. Voix et images du Crédif 2, 38-44.

Besse, H. (1984). Contexte(s) et enseignement / apprentissage d'une grammaire. Didactique des langues étrangères, 7-26.

Besse, H. \& Porquier, R. (1991). Grammaires et didactique des langues. Paris : CREDIF, HatierDidier.

Buchstaller, I. \& Khattab, G. (2013). Population samples. In Podesva R. J. \& Devyani, S. (eds). Research Methods in Linguistics, Cambridge : Cambridge University Press, 74-95.

Candelier, M. (dir.) (2003). Janua Linguarum - La Porte des Langues - L'introduction de l'éveil aux langues dans le curriculum. Strasbourg: Centre Européen pour les Langues Vivantes / Conseil de l'Europe.

Castellotti, V. (2001). La langue maternelle en classe de langue étrangère, Paris : CLE International.

Chaudron, C. (1983) Research on metalinguistic judgements: A review of theory, methods and results. Language Learning 33, 343-377.

Christidis, A.P. (1986). On the Modern Greek deictic particle na, Studies in Greek Linguistics 6. Proceedings of the $5^{\text {th }}$ annual conference on Greek linguistics, May 1985, 221-241, Thessaloniki : Kiriakidis.

Cowan, R. \& Hatasa, Y.A. (1994). Investigating the validity and reliability of native speaker and second-language learner judgments about sentences. In Tarone, E., Gass, S.M. \& Cohen, A. D. (eds). Research Methodology in Second Language Acquisition. Hillsdale, NJ : Lawrence Erlbaum, 287-302.

Ellis, R. (1991) Grammaticality judgements and second language acquisition. Studies in Second Language Acquisition 13,161-186.

Farrokhi, F. \& Mahmoudi-Hamidabad, A. (2012). Rethinking Convenience Sampling : Defining Quality Criteria. Theory and Practice in Language Studies, 2 (4), 784-792.

Fouillet, R., Stratilaki-Klein, S. \& Weber, C. (2015). Questionner les grammaires du français langue étrangère produites en contexte allophone. Le Français dans le monde. Recherches et applications. Paris : Français dans le monde : La grammaire en FLE/FLES. Quels savoirs pour quels enseignements ?, 38-46.

Holton, D., Mackridge, P. \& Philippaki-Warburton, I. (1997). Greek: a comprehensive grammar of the Modern Greek Language. London : Routledge.

Johnson, K. \& Johnson, H. (eds). (1998). Encyclopedic Dictionary of Applied Linguistics - A Handbook for Language Teaching. Oxford : Blackwell. 
Kalmbach, J.-M. (2008). Intégrer les marqueurs d'infinitif dans la grammaire française, Synergies Pays Scandinaves, 63-74.

Klairis, C. \& Babiniotis, G. (1999). Grammar of Modern Greek. Athens: Ellinika Grammata.

Mackay, A \& Gass, S. M. (2005). Second Language Research - Methodology and Design. Mahwah, NJ : Lawrence Erlbaum Associates.

Mackridge, P. (1985). The Modern Greek language. Oxford: Oxford University Press.

Monville-Burston, M. (2008). Évaluer la compétence par un test de jugement de grammaticalité : le cas de la relativisation chez des apprenants chypriotes hellénophones avancés en FLE. In Durand, J., Habert, B. \& Laks, B. (éds.) Congrès Mondial de Linguistique Française - CMLF'08, 17911803.

Monville-Burston, M. \& Kakoyianni-Doa, F. (2017). Les complétives en FLE : apprenants et enseignants chypriotes. Communication présentée à la journée d'étude Les descriptions contextualisées de la grammaire du français : État des recherches et perspectives. Sorbonne nouvelle, Département DFLE, 13-14 octobre 2017.

Philippaki-Warburton, I. (1994). The subjunctive mood and the syntactic status of the particle $n a$ in Modern Greek. Folia Linguistica. Societas Linguistica Europaea, 28 (3-4), 299-328.

Riegel, M., Pellat, J.-C. \& Rioul, R. (2016). Grammaire méthodique du français. Paris : PUF.

Sandfeld, K. (1965). Syntaxe du français contemporain. 3. L'infinitif. Genève : Droz.

Tremblay, A. (2005). Theoretical and methodological perspectives on the use of grammaticality judgment tasks in linguistic theory. Second Language Studies, 24(1), 129-167.

Trévise, A. (1996). Réflexion, réflexivité et acquisition des langues. AILE 8. Activités et représentations métalinguistiques dans les acquisitions des langues, 5-39.

\footnotetext{
${ }^{\mathrm{i}}$ Pour la question complexe de la thématisation en grec moderne, voir par exemple Mackridge 1985,
} Holton et al. 1997. 